\title{
Brian E. Vick, The Congress of Vienna. Power and Politics after Napoleon, Cambridge, MA, London: Harvard University Press 2014, VIII, 436 S., \$ 45,00 [ISBN 978-0-674-72971-1] \\ Besprochen von Peter Andreas Popp: Fürstenfeldbruck, E-Mail: PeterPopp@bundeswehr.org \\ DOI 10.1515/mgzs-2016-0035
}

»Frauen sind doch bessere Diplomaten« lautete der Titel des ersten deutschen Farbfilms - einer Ufa-Produktion aus dem Jahre 1941. Die Handlung dieser "volkstümlichen Komödie« mit Marika Rökk und Willy Fritsch in den Hauptrollen spielt im Revolutionsjahr 1848, dem Epochenjahr, das die Zeit des Biedermeiers beendete. Das hier vorzustellende Buch von Brian E. Vick mit dem Titel »The Congress of Vienna. Power and Politics after Napoleon« befasst sich hingegen mit dem diplomatischen Großereignis, das - auf deutsche Verhältnisse bezogen - die Zeit des Biedermeiers einleitete. Und um Frauen als bessere Diplomaten geht es auch in diesem Buch, genauer um deren kommunikative Rolle als Gastgeberinnen intellektuellen und politischen Personals (nebenbei bemerkt: es waren nur Männer!) in dem Ambiente, das dem Wiener Kongress sein eigentliches Gepräge gab: den Bällen und den Salons.

Wo findet Politik statt? Hinter den verschlossenen Türen der geheimen Kabinette und/oder auf dem öffentlichen Markt? Das ist hier die Frage. Um es vorweg zu nehmen: Vick ist mit seiner detailreichen und elegant geschriebenen Studie über die Inhalte, sprich Ergebnisse, des Wiener Kongresses sowie dessen gesellschaftliche Umstände ein wirklich großer Wurf gelungen. Er widerlegt das Bonmot »Le congrès danse beaucoup, mais il ne marche pas «, als dessen Urheber der effizienzorientierte, da aufklärerisch gesonnene österreichische Feldmarschall Charles Joseph de Ligne gilt; verstorben am 13. Dezember 1814 in Wien, also zu dem Zeitpunkt, als der Kongress wegen der polnisch-sächsischen Frage kurz vor dem Eklat stand. 
Verglichen mit bisherigen großen Studien über den Wiener Kongress wählt Vick einen für die Diplomatiegeschichte eher ungewöhnlichen Weg: Die ersten drei Kapitel des Werkes (»Peace and Power in Display«, »Selling the congress» und »Salon Networks«) gehen hinsichtlich des politisch-kulturellen Umfelds des Kongresses gleich in medias res. Kapitel 4, betitelt »Negotiating Religion«, widmet sich der Werteordnung und dem Wertegeflecht des vielleicht größten Friedenskongresses aller Zeiten. Der Wiener Kongress markierte nicht allein den Versuch, das von einem "großen kleinen Mann« (Napoleon) angerichtete Chaos nach dem Grundsatz "gerecht und billig « $\mathrm{zu}$ bewältigen, er bedeutete einen Einschnitt für die seit Ende des Mittelalters und damit seit Entstehung des internationalen Systems stattfindende Säkularisierung der Welt. Gemünzt auf die internationale Ordnung verkörpert der Wiener Kongress nach Vicks Auffassung den entscheidenden Schritt in Richtung auf eine bessere, weil im Zeichen der Aufklärung stehende Welt.

Die Tragik liegt darin, dass das im Zeichen des technischen Fortschritts schwelgende »lange« neunzehnte Jahrhundert die Lehren des Wiener Kongresses nicht beachten wollte. Das Kapitel »Negotiating Religion« nimmt die Schlüsselstellung innerhalb des Werkes ein. Markant ist hierbei auch der Raum, den der Autor der jüdischen Emanzipation auf staatsrechtlicher Ebene eingeräumt hat. Wann wirkt Emanzipation destabilisierend? Vick zeigt anhand dieses Sujets auf, welche Brisanz Ideen zukommt, die im öffentlichen Raum zunächst diskutiert, sodann in konkrete Politik umgesetzt werden sollen: Die mit der Spätaufklärung, der Französischen Revolution und der Napoleonischen Ära beförderte, aber in ihrer Totalität nach dem Wiener Kongress sogleich verhinderte jüdische Emanzipation wertet er als Parallelprozess zur Formierung des Nationalismus in Europa oder - wie man vielleicht besser sagen sollte - der Formierung der europäischen Nationalismen.

Während die ersten drei Kapitel sozial- und mentalitätsgeschichtlich orientiert sind, präferiert der Autor in Kapitel 4 die ideengeschichtliche Perspektive. Mit einem derart beharkten Feld gelingt umso besser die Einbettung der »klassischen«, d.h. diplomatiegeschichtlich orientierten letzten drei Kapitel mit den Überschriften »Europe in the Wider World«, »Between Reaction and Reform « und schließlich »Poland, Saxony, and the Crucible of Diplomacy«. Bisherige Gesamtdarstellungen des Wiener Kongresses führten die hier behandelten Sujets - die außereuropäische Dimension des Kongresses, verdeutlicht anhand der Frage des Sklavenhandels, kommt wahrlich nicht zu kurz! - immer an erster Stelle auf. Hier stehen sie im letzten Drittel.

Das ist alles andere als von Nachteil. Denn die »facts and figures« bedürften gerade in ihrer »Unvollkommenheit« des Exposés. Es ist nicht von der Hand zu weisen: Vick inszeniert seine Darstellung so, wie die österreichische Politik den 
Wiener Kongress inszenieren wollte: wohlproportioniert; nichts läuft aus dem Ruder. Jedes Kapitel umfasst ziemlich genau 40 bis 45 Seiten, in jedem wird die hohe Bedeutung des Faktors »Kommunikation« eigens hervorgehoben. Für den »eiligen Leser« hat das den großen Vorteil, dass jedes Kapitel für sich gelesen werden kann. Ob sich ihm auf diese Weise die Brillanz, d.h. Multiperspektivität des Werkes erschließt, sei dahingestellt: Das Buch, gelesen in einem Zug, enthält zugegebenermaßen gewisse Wiederholungen, so als nehme der Autor gerade ob der Dichte des historischen Ereignisses - oder sollte man angesichts der Verlaufsgeschichte des Kongresses nicht doch eher von komplexer Prozesshaftigkeit sprechen? - immer wieder den Faden für sich selbst auf, um dem Leser die Wertigkeit der »Öffentlichkeit« gegenüber »versuchter Geheimdiplomatie« vor Augen zu führen.

Vick zufolge war das gesellschaftliche Umfeld des Kongresses weder »Schnickschnack« noch schmückendes Beiwerk. Im Gegenteil: Die Inszenierung von Macht und der Faktor »Kommunikation«, praktiziert in jenen Salons, die ihrerseits als partieller Ersatz für eine noch nicht so wie in der westlichen Welt des 20. Jahrhunderts praktizierte Pressefreiheit gelten, waren genauso wichtig, ja fast noch wichtiger als die bloßen Verhandlungen des Kongresses. Für Vick steht außer Zweifel: Der Wiener Kongress ist in der publizistischen Nachwelt und auch in der historischen Forschung weithin einseitig beurteilt worden, weil diese sich auf die Jahre zwischen Waterloo und Krimkrieg (1815-1855/56) konzentriert haben, unter Vernachlässigung der unbedingt gebotenen Perspektive auf die Monate zwischen Herbst 1814 und Frühsommer 1815. Und so wirkt seine plastische Darstellung des Geschehens korrigierend gemäß dem Diktum Leopold Rankes, das jedes Ereignis unmittelbar zu Gott stehe.

Gerade der Wiener Kongress markiert - Vick nimmt auf den Sozialphilosophen Jürgen Habermas bewusst Bezug - den »Strukturwandel der Öffentlichkeit«, der mit der Aufklärung eingesetzt habe und mehr denn je heutiges westliches Politikverständnis präge - so die versteckte Botschaft des Autors und seines fulminanten Werkes -, wenn internationale Politik dem Kriterium der Humanität dienen will und soll. Doch läuft Vick bei der fast als Widmung zu verstehenden Betrachtung des Faktors »Öffentlichkeit« nicht Gefahr, vor lauter Öffentlichkeit einen »herrschaftsfreien Dialog « im Wien der Monate 1814/15 zu konstruieren, der so eben nicht gegeben war? Gerade hier sei realpolitisch vor Euphorie gewarnt: Das Medium »Öffentlichkeit«, konkret der Salon der Fanny von Arnstein, ist nicht $\mathrm{zu}$ verwechseln mit der politischen Botschaft des Kongresses an die unmittelbare Nachwelt in Form der Wiener Schlussakte. Das gesprochene Wort wirkt eben allzu oft wie »Schall und Rauch«. Was blieb, war eben das schriftlich fixierte Konferenzergebnis - so konkret oder so vage es wegen seiner Ausdeutbarkeit auch sein mochte. 
Doch für den Leser des Werkes dürfte - so steht zu hoffen - nach der Lektüre eines klarer denn je sein: Bei jedem historischen Ereignis kommen in Gesprächen formulierte Gedanken vor dem Ergebnis. Es war demnach gut, dass der Kongress tanzte. Anderenfalls wäre er abgebrochen worden. Herrschaftstechnisch betrachtet sollte der Historiker jedenfalls nicht den Faktor »Öffentlichkeit« vernachlässigen. Er konstituiert den Aggregatzustand, in dem Politik stattfindet - oder eben auch nicht. Vicks Darstellung des Wiener Kongresses schärft dafür den Blick. Und abschließend sei bemerkt: Es hatte herrschaftstechnisch betrachtet schon seinen Sinn, wenn der Metternichsche Polizeiapparat bemüht war, alles aufzuschreiben. Davon nämlich profitiert Vicks Darstellung. Doch ob die zeitgenössischen Autoren tatsächlich alles erfassten, wie der Historiker es gerne erfasst haben wollte, steht auf einem anderen Blatt. 